cases in the Netherlands occur in men who have sex with men (MSM). Not all infections result in clinical symptoms and not all persons with clinical symptoms are diagnosed. We performed a study among MSM visiting the STI clinic in Amsterdam to assess the prevalence of Shigella.

Methods From March to June, 2020, Anal swab samples taken from MSM routinely visiting the STI clinic to detect Chlamydia trachomatis and Neisseria gonorrhoeae were additionally tested pseudonymously for the presence of Shigella by PCR on the ipaH gene. Consecutive samples from MSM who reported no diarrhea, or diarrhea during last month, or diarrhea at visit of clinic were included. Predefined minimal numbers of inclusion of these groups were 150, 100 and 50, respectively. During the same months the frequency of Shigella as assessed by PCR in routinely tested samples sent by general physicians was assessed.

Results We included samples from 214 MSM without diarrhea, 109 MSM who recently had diarrhea and 68 MSM who reported diarrhea at visit of the clinic. The total number of samples positive for Shigella was $13 / 389$ (3.3\%), of whom 6/ $212(2.8 \%)$ had no diarrhea, 4/107 (3.7\%) recently had diarrhea and $3 / 68(4.4 \%)$ had diarrhea at clinic visit. Positive samples were more frequently found in persons using or recently having used PREP (10/152), compared to no PREP (2/163) or being HIV-positive $(1 / 74)$ ( $p=0.02$, chi square test). In comparison, only $11 / 774(1.4 \%)$ routinely tested fecal samples sent by general physicians during the study period were positive for Shigella.

Conclusion Shigella infections without symptoms or with minor symptoms are relatively common in MSM. More detailed studies should focus on the risk of transmission from these persons to others, leading to symptomatic infections.

\section{P319 PHARMACODYNAMICS OF CEFTRIAXONE, ERTAPENEM, FOSFOMYCIN AND GENTAMICIN IN NEISSERIA GONORRHOEAE}

${ }^{1} \mathrm{~A}$ Van Dam*, ${ }^{2} \mathrm{M}$ De Laat, ${ }^{3,4} \mathrm{~A}$ Boyd, ${ }^{1,5} \mathrm{~A}$ Van Dam. ${ }^{1}$ Public Health Laboratory, Amsterdam Health Service, Amsterdam, The Netherlands; ${ }^{2}$ STI Clinic, Amsterdam Health Service, Amsterdam, The Netherlands; ${ }^{3}$ Stichting HIV monitoring, Amsterdam, The Netherlands; ${ }^{4}$ Department of Research, Amsterdam Health Service, Amsterdam, The Netherlands; ${ }^{5}$ Medical Microbiology, Amsterdam UMC, Amsterdam, The Netherlands

\subsection{6/sextrans-2021-sti.374}

Introduction Due to ceftriaxone-resistance in Neisseria gonorrhoeae, the use of other antibiotics might become necessary for treatment. In the recently performed NABOGO trial, efficacy of ertapenem, gentamicin and phosphomycin was compared with ceftriaxone. In this in vitro study, the pharmacodynamics of these antibiotics were studied.

Methods The ceftriaxone-susceptible N. gonorrhoeae strain WHO-F, the ceftriaxone-resistant strain WHO-X and the clinical strain CS03307 were used. Standard MICs for study antibiotics were measured by e-tests. Using time-kill assays for each antibiotic and strain, time-kill curves were constructed by measuring bacterial growth rates at doubling antimicrobial concentrations. Maximal growth in the absence of antibiotics ( $\psi \max )$, minimal (negative) growth in the presence of antibiotics ( $\psi \mathrm{min})$, and concentration of antibiotic resulting in stationary amounts of CFU/ml (zMIC) were calculated.

Results $\psi \max$ values were between 0.5 and 0.85 and did not vary between strains. $\psi$ min values for ceftriaxone were -2.7 for the highly susceptible WHO-F strain (MIC $=<0.002 \mathrm{mg}$ /
1), but only -0.9 for $\mathrm{CS} 03307$ (MIC $=0.012 \mathrm{mg} / \mathrm{l}$ ), and -0.8 for the resistant WHO-X strain $(\mathrm{MIC}=1.5 \mathrm{mg} / \mathrm{l}) . \psi$ min values for ertapenem were -1.1 for WHO F $(\mathrm{MIC} \leq 0.002 \mathrm{mg} / \mathrm{l}),-0.9$ for $\mathrm{CS} 03307 \quad(\mathrm{MIC}=0.006 \mathrm{mg} / \mathrm{l})$, and -0.5 for WHO-X $(\mathrm{MIC}=0.032 \mathrm{mg} / \mathrm{l})$, reflecting slower bacterial killing than ceftriaxone. All strains were rapidly killed by gentamicin as shown by $\psi$ min values of -5 to -7 (range of $\mathrm{MIC}=1.5-3 \mathrm{mg}$ / 1). Phosphomycin $\psi$ min values were -1.7 for WHO-F $(\mathrm{MIC}=24 \mathrm{mg} / \mathrm{l})$ and CS03307 (MIC=4 mg/l), but only -0.5 for WHO-X $(\mathrm{MIC}=12 \mathrm{mg} / \mathrm{l})$. For ceftriaxone, zMICs were three- to sixfold lower than MICs, but for other antibiotics, zMICs were comparable to MICs.

Conclusion Compared to ceftriaxone, bacterial killing of ertapenem is less rapid and is affected in WHO-X despite low MIC. Given the differences observed between zMIC and MIC, ceftriaxone might not be completely ineffective in ceftriaxoneresistant strains, especially if given in higher and repeated dosages.

\section{P320 SEXUAL BEHAVIOURS AND HERPES SIMPLEX VIRUS TYPE-2 INCIDENCE AND PREVALENCE AMONG ADOLESCENT GIRLS AND YOUNG WOMEN IN KWAZULU-NATAL, SOUTH AFRICA}

${ }^{1} \mathrm{~W}$ Fong ${ }^{*},{ }^{2} \mathrm{~N}$ Mthiyane, ${ }^{1,2,3,4,5} \mathrm{G}$ Harling, ${ }^{2} \mathrm{~N}$ Okesola, ${ }^{2} \mathrm{O}$ Behuhuma, ${ }^{1,2} \mathrm{~N}$ Chimbindi, 1,2,3 T Zuma, ${ }^{2} \mathrm{~J}$ Dreyer, ${ }^{2} \mathrm{C}$ Herbst, ${ }^{2} \mathrm{~T}$ Smit, ${ }^{2} \mathrm{~S}$ Danaviah, ${ }^{1} \mathrm{~L}$ Sherr, ${ }^{2,3,6} \mathrm{~J}$ Seeley, ${ }^{6} \mathrm{~S}$ Floyd, ${ }^{6}$ Birdthistle, ${ }^{1,2,3} \mathrm{M}$ Shahmanesh, ${ }^{1} \mathrm{P}$ Sonnenberg. ${ }^{1}$ Institute for Global Health, University College London, London, UK; ${ }^{2}$ Africa Health Research Institute, KwaZulu-Natal, South Africa; ${ }^{3}$ University of KwaZulu-Natal, KwaZulu-Natal, South Africa; ${ }^{4} \mathrm{MRC}$ Wits Rural Public Health and Health Transitions Research Unit (Agincourt), University of the Witwatersrand, Gauteng, South Africa; ${ }^{5}$ Department of Epidemiology and Harvard Centre for Population and Development Studies, Harvard T.H. Chan School of Public Health, Boston, USA; ${ }^{6}$ London School of Hygiene and Tropical Medicine, London, UK

\subsection{6/sextrans-2021-sti.375}

Background Adolescent girls and young women (AGYW) in rural KwaZulu-Natal, South Africa are disproportionately affected by herpes simplex virus type 2 (HSV-2) and HIV. As HSV-2 infection enhances the acquisition and transmission of HIV and other STIs, broader STI control requires understanding of HSV-2 predictors. We therefore assessed how HSV-2 incidence and prevalence were associated with sexual behaviours among AGYW in this region.

Methods We analysed data from a two-year (2017-2019) prospective cohort study among a random sample of 2184 AGYW aged 13-22 which was representative of the study population. Data were collected at baseline, 12 months and 24 months. We calculated HSV-2 prevalence at any study visit and incidence among those HSV-2 negative at baseline. We ran Poisson and logistic regressions to assess the association of sexual behaviours with both HSV-2 incidence and prevalence.

Results HSV-2 prevalence was $26.2 \%$ among the study population, and was strongly associated with having sexual debut before the age of 16 (adjusted odds ratio (aOR) 1.52, 95\% confidence interval (CI) 1.06-2.17) and having two or more sexual partners in the past 12 months (aOR 3.64, 95\% CI 1.39-9.50). 307 of 1,433 baseline HSV-2 seronegative AGYW seroconverted during follow-up (incidence rate 16.5/100 person years at risk, 95\% CI 14.7-18.4). Self-reported lower age of sexual debut, one or more sexual partners and inconsistent condom use were bivariately associated with HSV-2 incidence. None of these self-reported sexual behaviour measures 